\title{
Dermatologic Toxicities from Monoclonal Antibodies and Tyrosine Kinase Inhibitors against EGFR: Pathophysiology and Management
}

\author{
Shaad E. Abdullah, Missak Haigentz Jr., and Bilal Piperdi \\ Division of Oncology, Department of Medicine, Montefiore Medical Center, Albert Einstein College of Medicine, Bronx, \\ New york, NY 10461, USA \\ Correspondence should be addressed to Bilal Piperdi, bpiperdi@montefiore.org
}

Received 18 May 2012; Revised 24 July 2012; Accepted 24 July 2012

Academic Editor: Sue S. Yom

Copyright ( $) 2012$ Shaad E. Abdullah et al. This is an open access article distributed under the Creative Commons Attribution License, which permits unrestricted use, distribution, and reproduction in any medium, provided the original work is properly cited.

Epidermal growth factor receptor (EGFR) inhibition has now been well established as an effective treatment for various cancers. The EGFR belongs to the ErbB family of tyrosine kinase receptors which regulate tumor cell differentiation, survival and proliferation. Activation of EGFR drives tumorigenesis in lung, head and neck, colorectal and pancreatic cancers. Irrespective of the type of cancer being treated and the mechanism by which tumor EGFR drives tumorigenesis, the major side effect of EGFR inhibition is a papulopustular (also described as maculopapular or acneiform) rash which occurs in about two thirds of treated patients. Interestingly, this rash has been commonly correlated with better clinical outcomes (objective tumor response and patient survival). The pathophysiology of dermatological toxicity from EGFR inhibitors is an important area of clinical research, and the proper management of the rash is essential to increase the therapeutic index from this class of drugs. In this paper, we review the dermatologic toxicities associated with EGFR inhibitors with an emphasis on its pathophysiology and clinical management.

\section{Introduction}

Epidermal growth factor receptor (EGFR) inhibition has now been well established as an effective treatment for various cancers. EGFR belongs to a family (ErbB) of tyrosine kinase receptors which regulate tumor cell differentiation, survival, and proliferation. EGFR drives tumorigenesis as a result of activating mutations in adenocarcinoma of the lung and by less defined mechanisms of pathway activation (increased expression of receptors or ligands) in other malignancies such as head and neck cancer, colorectal cancer, squamous cell carcinoma of the lung, and pancreatic cancer [1].Best responses and clinical benefit have been seen in malignancies with EGFR activating mutations but clinical benefit has also been observed in conditions where the pathway is not activated as a result of EGFR mutations.

Irrespective of the type of cancer being treated and the mechanism by which tumor EGFR drives tumorigenesis, the major side effect of EGFR inhibition is a papulopustular (also described as maculopapular or acneiform) rash which occurs [1] in about two thirds of the patients. When severe (grade 3 , in about $10 \%$ of the patients), it often leads to treatment discontinuation. In a larger number of patients, it affects quality of life affecting compliance and often results in treatment dose adjustments or temporary interruptions [2-4]. Different reports suggest that dose modifications or interruptions as a result of skin toxicity occur as often as about $30 \%$ of patients $[5,6]$. Understanding the pathophysiology and management of dermatological toxicity from EGFR inhibitors is an important area of clinical research, and the proper management of the rash is essential to increase the therapeutic index from this class of drugs. There is no general consensus regarding the treatment of the rash. Several recent trials have evaluated empiric interventions and attempts have been made to establish guidelines [710]. Interestingly, when the relationship has been studied, the rash has been uniformly correlated with better clinical outcomes (objective tumor response and patient survival) 
both when the anti-EGFR agents are used as single agents or in combination with chemotherapy [11-16]. In this paper, we will review the dermatologic toxicities associated with EGFR inhibitors with emphasis on pathophysiology of the rash and its management.

\section{Epidermal Growth Factor Receptor and Pathway}

The erbB oncogenes encode the HER family of tyrosine kinase receptors, which namely consists of EGFR or HER1, HER2, HER3, and HER4. All members of the HER family consist of a receptor which comprises of an extracellular site concerned with ligand binding, a hydrophobic transmembrane domain, and an intracellular tyrosine kinase domain. Ligands binding to the EGFR are namely the epidermal growth factor (EGF), amphiregulin, $\beta$-cellulin, epiregulin, Heparin-binding EGF-like ligand (HB-EGF), transforming growth factor alpha (TGF $\alpha)$, and others. Binding of the ligand to the extracellular domain of the receptor results in conformational change and subsequent dimerization of the receptor with other HER family receptors. After dimerization, the internal tyrosine kinase is activated resulting in a downstream signaling cascade involving various pathways such as the PI3K/AKT, Ras/Raf/MAPK, and so forth. These activated signaling pathways result in interaction with essential cellular processes such as inhibition of apoptosis, gene transcription/translation, cell cycle progression, proliferation, and even processes involved with glucose metabolism $[17,18]$.

\section{EGFR Inhibitors and Cancer}

The EGFR is expressed in many different cell types in normal tissues, such as epithelial tissue, skin, hair follicles, and the gastrointestinal tract. In malignancy, however, dysregulation or overexpression of the receptor can occur leading to evasion from apoptosis, proliferation, invasion, metastases, and tumor induced angiogenesis [19-21]. Treatment options have become available over the years which inhibit the receptor or the downstream pathway, either by using monoclonal antibodies (MAbs) binding to the extracellular domain, small molecule tyrosine kinase inhibitors (TKIs) which bind to the intracellular domain, antisense oligonucleotides which decrease the expression of EGFR, immunotoxins which deliver toxins by targeting the receptor, and direct inhibitors of downstream signaling. The first EGFR inhibitor (EGFRI) to become widely available for clinical use was cetuximab (Erbitux), a chimeric human/murine immunoglobulin (IgG1) monoclonal antibody which binds to EGFR which higher affinity than EGF or TGF $\alpha$ [22]. Cetuximab was first approved by the US FDA in 2004 in combination with irinotecan or as a single agent in patients unable to tolerate irinotecan for colorectal cancer. In 2006, cetuximab was approved for the treatment of squamous cell carcinoma of the head and neck in combination with radiation therapy or as a single agent in patients who had received cisplatin previously, while another monoclonal but fully humanized antibody panitumumab was approved for colorectal cancer in 2007 for metastatic disease. Available small molecule EGFR tyrosine kinase inhibitors are gefitinib (Iressa) and erlotinib (Tarceva) for patients with metastatic lung cancer.

\section{EGFRI-Associated Rash and Pathophysiology}

Dermatologic toxicities are the most common side effects associated with anti-EGFR therapy. The most common dermatologic toxicity resulting from EGFRI treatment is papulopustular eruption, also called acneiform rash. Additional toxicities include nail changes, hair changes, ocular changes, pruritis, xerosis, and photosensitivity or erythema. EGFR inhibitor-related rash occurs very frequently in the prescribed patients, usually starting within two to three days following initiation of EGFRI treatment, and worsen within one to three weeks. Although not life threatening, the rash can be significant in causing impairment in quality of life. Not only can the rash cause discomfort but also occur in areas such as the face were it can be cosmetically and emotionally detrimental for the patient.

EGFR protein is normally expressed in a wide variety of tissues, including the normal epidermis and follicular keratinocytes in the basal layer, the outer root sheath of hair follicles, and various other cells of the dermal connective tissue system. Approximately 40,000 to 100,000 receptors are reported to be expressed on the cell surface of normal tissues [23], while the number can reach around 2 million receptors per cell in malignancy $[24,25]$. EGFR takes part in the various essential stages of epithelial maintenance by causing epidermal growth, differentiation, taking part in wound healing and keratinocyte migration. Hence, under EGFR inhibition, there is abnormal upkeep of the epithelium resulting in altered maturation and neutrophilic suppurative infiltrate in the dermis, particularly involving the follicular infundibula. The follicles are frequently enlarged in size and sometimes obstructed by excess keratinocyte plugs, while the sebaceous glands are usually not affected. No consistent changes in the cutaneous microflora have been found [26].

In animal models, inhibition of EGFR blocks downstreams signaling pathways and prevents keratinocytes from maturing properly as they migrate to the outer stratum corneum [27]. This results in the thinning of the outermost layers of the epidermis and corneal layers, and the subsequent loss of the skin's protective barrier function results in increased sensitivity to UV radiation damage.

The EGFRI-associated rash appears to be clinically more severe with the use of monoclonal antibodies compared to the EGFR tyrosine kinase inhibitors (Table 3) [28]. Of note, the severity of rash has not always been directly correlated with the degree of EGFR inhibition. In a set of studies in patients treated with erlotinib, the skin inflammatory reaction was found to be independent of the degree of EGFR inhibition, and the cellular response was found to be mediated by TRAIL-positive mononuclear cells initially, and later by polymorphonuclear cells when superinfection from the skin occurs [29]. There is very little information available regarding the clinical or molecular predictors of rash severity 
from EGFRI. Although EGFR activating mutations in tumors have been predictive for clinical benefit to EGFR targeting agents (specifically TKIs) [30], these somatic mutations are present in only the tumor and therefore cannot account for the positive association of skin rash and clinical benefit with these agents. Research attention has recently focused on EGFR gene polymorphisms, present in both skin and tumor tissues. Various pharmacodynamic and pharmacogenomics models have been studied where EGFR polymorphisms have positively correlated with appearance of skin rash in patients, including downstream polymorphisms such as of the PI3 kinase pathway [31-34]. It has been postulated that these polymorphisms play a role in the positive overall survival correlation of the skin rash. The host susceptibility to respond in the form of an inflammatory reaction to EGFR inhibition may play an important role in the pathogenesis of EGFRI-associated rash. The role of host immune system in the pathogenesis of EGFRI-related dermatologic toxicities is poorly understood.

The combination of these inflammatory events leads to the eruption of varying degrees from mild to severe with complicated superinfection since the innate barrier system of the skin is compromised. The skin can become sensitive to UV light, while pruritus, discomfort, irritation, skin flaking and, associated hail and nail changes severely hamper patient quality of life. Emotional distress and also body image issues can also severely impact the patients' day-to-day life and compliance to therapy [35].

Interestingly, recent results from clinical trials involving nimotuzumab, a new anti-EGFR monoclonal antibody, have reported to have lesser or no skin toxicity which has been postulated to be secondary to selective inhibition for tumor cell EGFR phosphorylation only while sparing the skin [3638].

\section{Grading of EGFRI-Associated Rash}

It is important to follow the patient closely after initiation of EGFR inhibitor therapy for the development of rash which usually develops at the first 1-2 weeks, peaks at 3-4 weeks on therapy, and then most of the time diminishes in intensity over the next couple of weeks but often persists in mild form throughout the course of therapy. Several grading criteria have been developed to judge the severity of rash. The primary goals of these grading criteria have been to develop a uniform, common terminology for assessment of rash severity and to help clinicians tailor therapy depending on the severity of the rash.

The National Cancer Institute Common Toxicity Criteria for Adverse Events (CTCAE), version 4.0, allows for a quick severity estimation of skin toxicity reactions. It provides a clinical score with five grades of severity but heavily relies on the body surface area distribution which is not specific to EGFRI-associated rash, which is often limited to the face, scalp, chest, and upper back. Similarly, the sensitive dermatological skin score (WoMoScore) focuses on the long-term assessment of acneiform skin rashes [39]. The WoMoScore is calculated from body involvement, facial involvement, and clinical grading of erythema, papulation, pustulation, scaling, and crusts, providing a clinical score ranging from 0 to 100 (Table 4). Mild skin changes score up to 20 , moderate cases range between 20 and 40 , whereas severe acneiform eruptions exceed a WoMoScore of 40 . However, this scoring system also is not entirely specific to EGFRI-associated rash and is not always practical for routine use by clinicians and oncologists.

Two other EGFRI-relevant grading systems have been proposed, the first by Pérez-Soler et al. in 2005 [40] (Table 1) which accurately allows physicians to tailor EGFRI therapy and to treat the rash proactively. Another classspecific grading system has been adapted recently by the Multinational Association of Supportive Care (MASCC skin toxicity group; Table 2) which also is helpful to the healthcare team in accurately grading the toxicity of EGFRI [41].

\section{Other Dermatologic Toxicities Observed with EGFRI Therapy}

In addition to the common side effect of rash, therapy with EGFR inhibitors can also be associated with other dermatologic toxicities. These include nail, hair, and ocular changes, as well as xerosis, pruritis, and photosensitivity. Recognizing these side effects and educating, monitoring, and providing supportive care to patients will promote better compliance with the treatment and will help the patients with coping strategies for these toxicities.

6.1. Xerosis, Pruritis, and Photosensitivity. Patients on EGFRI therapy can have a scaly, dry, pruritic skin in varying degrees in any part of the body including ocular, perineal, and vaginal areas. Xerosis is defined as dry, flaking skin and is seen in about $35 \%$ of patients treated with EGFRIs. Pruritis is defined as an unpleasant sensation that leads to itching of the skin and occurs in response to the release of histamine. Although the xerosis and pruritis tend to occur together, some patients may experience dry skin only without pruritus. Itching as a result of dry skin and pruritis can often lead to superinfection, resulting in cellulitis and folliculitis. Xerosis can also lead to swelling and cracking of lips, mucosal irritation, erythema, and inflammation [42]. Patients treated with EGFRIs may also develop varying degrees of photosensitivity for which the mechanism is not currently understood. EGFRI-induced photosensitivity is usually characterized by erythema from UV-induced damage. Erythema may be painless or painful and associated with mild to severe desquamation [41].

6.2. Hair Changes. Alopecia and hirsutism have been reported with the use of EGFRI therapy. Usually, alopecia develops 2-4 months after initiation of EGFRI therapy in $5-6 \%$ of the patients, while hirsutism can occur after 12 months on EGFRI therapy. Hirsutism is usually characterized by causing hair curling and rigidity, facial hypertrichosis, and trichomegaly. The latter involving the eyelashes can lead to eye irritation and conjunctivitis $[41,43,44]$. 
TABle 1: Parmar et al. modified EGFRI rash grading system [31].

\begin{tabular}{ll}
\hline Grade 1 & $\begin{array}{l}\text { Macular or papular rash or erythema } \\
\text { but no other associated symptoms }\end{array}$ \\
\hline
\end{tabular}

Grade 2A

Grade $1+$ pruritis or other symptoms which are tolerable

Grade 2

Grade 2B

Grade + pruritis or other symptoms which interfere with daily life

Grade 3

Severe, generalised erythroderma, or macular, popular, or vesicular eruption

Grade 4 Generalized exfoliative, ulcerative, or blistering skin toxicity

TABLE 2: MASCC skin toxicity group proposed rash staging (papulopustular eruption, grading individually for face, scalp, chest, or back).

\begin{tabular}{|c|c|}
\hline \multirow{2}{*}{ Grade 1} & $\begin{array}{l}\text { Grade 1A: papules or pustules }<5 \text {; OR } 1 \text { area of } \\
\text { erythema or edema }<1 \mathrm{~cm} \text { in size }\end{array}$ \\
\hline & $\begin{array}{l}\text { Grade 1B: papules or pustules }<5 \text {; OR } 1 \text { area of } \\
\text { erythema or edema }<1 \mathrm{~cm} \text { in size AND pain or } \\
\text { pruritis }\end{array}$ \\
\hline \multirow{2}{*}{ Grade 2} & $\begin{array}{l}\text { Grade } 2 \mathrm{~A} \text { : papules or pustules } 6-20 \text {; OR } 2-5 \text { areas of } \\
\text { erythema or edema }<1 \mathrm{~cm} \text { in size }\end{array}$ \\
\hline & $\begin{array}{l}\text { Grade } 2 \mathrm{~B} \text { : papules or pustules } 6-20 \text {; OR } 2-5 \text { areas of } \\
\text { erythema or edema }<1 \mathrm{~cm} \text { in size AND pain, } \\
\text { pruritus, or effect on emotions or functioning }\end{array}$ \\
\hline \multirow[b]{2}{*}{ Grade 3} & $\begin{array}{l}\text { Grade 3A: papules or pustules }>20 \text {; OR more than } 5 \\
\text { areas of erythema or edema }<1 \mathrm{~cm} \text { in size }\end{array}$ \\
\hline & $\begin{array}{l}\text { Grade 3B: papules or pustules }>20 \text {; OR more than } 5 \\
\text { areas of erythema or edema }<1 \mathrm{~cm} \text { in size; AND } \\
\text { pain, pruritus, or effect on emotions or functioning }\end{array}$ \\
\hline
\end{tabular}

6.3. Nail Changes. EGFRI therapy is also associated with paronychia which can occur in about $6-12 \%$ of patients and most commonly affects the nail bed of the great toe [45]. These nail changes lead to inflammation, tenderness, formation of pyogenic granuloma-type lesions, and fissuring of lateral nail folds or distal finger nail bed. When severe, nail extraction could be required. The nail changes usually develop after 2-3 months of therapy but can also be observed as late as 6 months on therapy and can persist a long time after the drug has been halted [42].

6.4. Ocular Changes. Corneal dryness and abrasions can occur on EGFRI therapy. Most ocular changes can occur early in the course of therapy, sometimes seen even in the first week or two of therapy and can significantly impair patient quality of life. The majority of ocular changes are secondary to involvement of the eyelids and include squamous blepharitis, trichomegaly, meibomitis, dysfunctional tear syndrome, and other miscellaneous changes such as iridocyclitis and corneal epithelial defects $[46,47]$.

\section{Management of EGFRI-Associated Rash and Skin Changes}

With increasing use of EGFR inhibitors in clinical practice, we will notice an increasing need to recognize dermatologic toxicities associated with these agents and understand their management. The proper evidence-based management of the dermatologic side effects will be of paramount importance to their optimal use across a variety of different cancers. The majority of the literature on managing EGFRI-related dermatologic toxicities includes descriptive and case studies describing skin treatment regimens for managing toxicities associated with EGFRI treatment. Several recent prospective studies have addressed and evaluated different interventions to mitigate or reduce the severity of EGFRI-associated skin rash. Though there are many avenues in terms of treating the side effects associated with EGFRI therapy they mainly are employed in two ways, either reactionary to treat the rash which has occurred or preemptive/prophylactically in advance to avoid progression to a stage which interferes with patients' quality of life and compliance with therapy. Attempts have also been made to develop a consensus on treating the rash associated with EGFRI [8, 9, 40, 48-51].

7.1. General Patient Recommendations. The proper patient education and understanding of the potential dermatological side effects from EGFRI is the essential cornerstone of its management. At the start of EGFRI treatment, the clinicians should inform their patients of potential EGFRIrelated symptoms of dermatologic toxicities and possible lifestyle changes that may enhance their comfort. Information regarding a patient's occupation and previous skin conditions and sensitivities may help clinicians and providers design treatment strategies for individual patients that can help prevent or minimize EGFRI-related dermatologic toxicities.

One such measure should be to advise avoidance of prolonged sun exposure. If sun exposure is expected while patient is on EGFRI therapy, then the use of sunscreen is recommended with an at least 15 sun protection factor (SPF) or higher cream which should be applied to exposed areas 1-2 hours prior to exposure and reapplied every 2 hours if out in the sun for an extended period [52]. Other general measures include use of moisturizing cream for dry skin, antihistamines for pruritis and proper nail care. Patients 
TABLE 3: Dermatologic toxicity reported with single agent EGFR inhibitor therapy.

\begin{tabular}{lcc}
\hline & Any grade $(\%)$ & Grade 3 and 4 (\%) \\
\hline Cetuximab [54, 55] & $80-86$ & $5-18$ \\
Panitumumab [56] & 90 & 14 \\
Erlotinib [11, 57] & $75-79$ & $5-10$ \\
Gefitinib [58] & $62-75$ & Up to 4 \\
Lapatinib [59] & 27 & 1 \\
\hline
\end{tabular}

TABle 4: Definition of the score items of the WoMoScore.

\begin{tabular}{ll}
\hline$A=$ body involvement & $\begin{array}{l}\text { Extent of body lesions, 0-100\%, } \\
\text { according to the rule of nines. }\end{array}$ \\
$B=$ facial involvement & $\begin{array}{l}\text { Extent of lesions in the face, } 0-100 \% \\
\text { Sum of: } \\
\text { erythema intensity }(0-3)\end{array}$ \\
& erythema distribution $(0-3)$ \\
& papulation $(0-3)$ \\
& pustulation $(0-3)$ \\
& scaling/crusts $(0-3)$ \\
\hline
\end{tabular}

Calculation formula for final WoMoScore: WoMoScore $=1 / 4 A+1 / 4 B+$ 10/3C.

should be instructed to apply moisturizing cream at least twice daily using a thick alcohol-free emollient. Patients should avoid products that lead to drying of the skin such as alcohol-based products, soaps, and long hot showers which can also dry the skin [53]. Patients should be instructed to avoid the common topical antiacne agents such benzoyl peroxide, which has the potential to cause skin irritation and excessive drying.

\section{Reactive Treatment Strategies for Skin Rash}

The majority of studies and interventions have evaluated several treatment strategies for skin rash after it has developed from anti-EGFRI therapy. Guidelines established have investigated various modes of treatment tailored to severity of rash incorporating topical and systemic antibiotics, topical and systemic steroids, and immunosuppressives.

8.1. Topical Treatment. Topical steroids and antibiotics have shown benefit in treating rash and preventing superinfection. Medium to high potency topical steroid preparations (e.g., hydrocortisone $1 \%$ or $2.5 \%$ ) have been employed to treat the rash after in vitro studies showed release of inflammatory cytokines following EGFRI therapy. The topical antibiotics commonly used are clindamycin, erythromycin, and metronidazole. European studies have suggested use of topical metronidazole and oral minocycline $100 \mathrm{mg}$ one to two times daily as a treatment for acneiform rash $[60,61]$.

8.2. Oral Antibiotics. Several studies thus far have reported benefit from the reactive use of oral tetracycline-based antibiotics. Recent guidelines established by the MASCC skin toxicity study group [62] have graded preparations to the level of evidence available for their use in EGFRI-associated rash. In these recommendations, doxycycline $100 \mathrm{mg}$ BID and minocycline $100 \mathrm{mg}$ daily have been advised for systemic use. Doxycycline is a preferred agent in patients with renal insufficiency, while minocycline is less photosensitizing.

In another study reported by Scope et al. from the Memorial Sloan Kettering Cancer Center, patients with metastatic colorectal cancer receiving cetuximab were randomly assigned to either oral minocycline with topical tazarotene (a retinoid that is part of the vitamin A family) or oral placebo with topical tazarotene to reduce or prevent cetuximab-related rash [7]. The primary endpoint was total facial lesion count. Forty-eight patients were randomly assigned to receive minocycline or oral placebo, along with daily tazarotene cream at the start of treatment. Results from the study indicated that during the first month of treatment, total facial lesion counts were less in patients who received minocycline, including less pruritus than the placebo arm (20\% versus $50 \%$, resp.; $P=0.05)$. Administration of tazarotene cream had no clinical benefit, and skin irritation was reported in one-third of patients. Results from this study have encouraged physicians and nurses to administer oral minocycline when treating patients with papulopustular eruptions associated with EGFRI treatment and to avoid use of retinoids.

In another case study by Shu et al., treatment with oral minocycline was effective in the treatment of nail changes or paronychia elicited by EGFRI use. In summary, studies indicate a reactionary role for tetracycline-based oral antibiotics in the reactive management of skin rash associated with EGFRI. These agents have proven their efficacy in decreasing the number and extent of papulopustular eruptions in randomized trials with the use of oral minocycline [7], tetracycline [63], and doxycycline [8]. General treatment recommendations are summarized in Figure 1, which outlines the treatment protocol with increasing rash severity.

\section{Preemptive or Prophylactic Rash Management}

The skin toxicity evaluation protocol with panitumumab study (STEPP) compared in a randomized prospective manner the preemptive skin treatment protocol versus a reactionary skin treatment protocol in patients who were receiving panitumumab in combination with chemotherapy in patients with advanced colorectal cancers [8]. Patients on the reactive arm received treatment after the rash developed, while the pre-emptive group was assigned to receive daily skin therapy for a total of 6 weeks, starting 24 hours prior to the first dose of the EGFR inhibitor, panitumumab. This preemptive treatment protocol included sunscreen, moisturizer, hydrocortisone $1 \%$ cream, and oral doxycycline at $100 \mathrm{mg}$ BID. When both arms were compared, the pre-emptive arm had a reduced incidence of grade 2 or greater rash by more than $50 \%$, without any additional adverse effects of added therapy. There was also a significant delay in grade 2 or greater rash first occurrence in the pre-emptive arm. Also the 
Rash severity

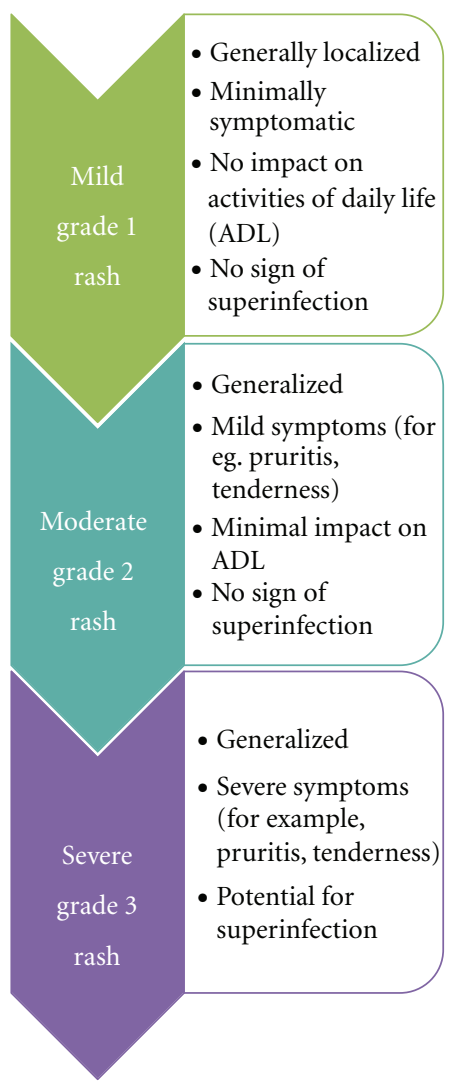

\section{Intervention}
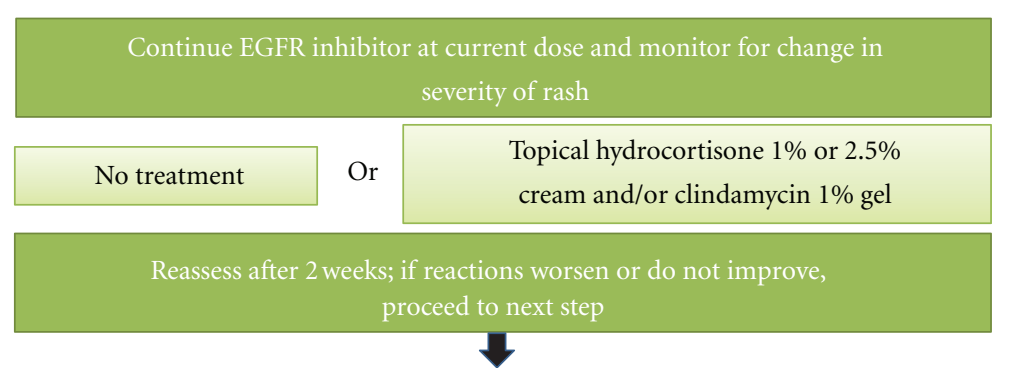

Continue EGFRI at current dose and monitor for change in severity; continue treatment of skin reaction with the following

Hydrocortisone $2.5 \%$ cream or clindamycin $1 \%$ gel or pimecrolimus $1 \%$ cream plus doxycycline $100 \mathrm{mg}$ PO BID or minocycline $100 \mathrm{mg}$ POBID

Reassess after 2 weeks; If reactions worsen or do not improve, proceed to next step $+$

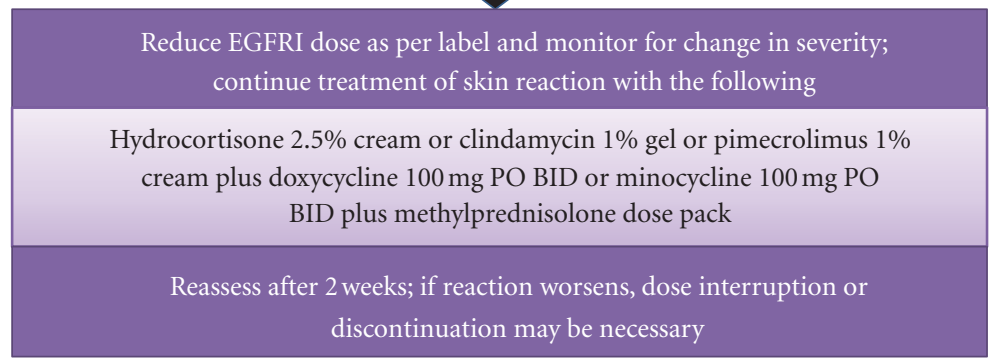

FIGURE 1: Treatment recommendations for EGFRI-associated rash. Adapted from Lynch et al. 2007 [45].

Dermatology Life Quality Index (DLQI) showed that patient quality of life was less impaired in the pre-emptive arm. The median overall and progression-free survival was similar between the two groups, indicating that the preemptive treatment of skin rash had no adverse impact on the outcome from anti-EGFR therapy. This study makes a strong case of pre-emptive treatment of the rash but the use of multiple agents in the pre-emptive arm makes it difficult to assess which one of the pre-emptive treatments was most effective in creating this difference.

Although a study reported by the researchers from the North Central Cancer Treatment Group, the role of oral tetracycline was examined in reducing the incidence of EGFRI-related rash $[63,64]$. A total of 61 patients who received various anti-EGFR therapies were randomly assigned to receive either oral tetracycline $(n=31 ; 500 \mathrm{mg}$ orally twice per day for 28 days) or placebo $(n=30)$. Tetracycline did not decrease the incidence of rash compared with placebo $(70 \%$ and $76 \%$ of patients in the tetracycline and the placebo arm, respectively, developed papulo-pustular eruption). However, the severity of rash was significantly lower in patients receiving tetracycline. After four weeks of treatment, $17 \%$ of patients treated with tetracycline reported grade 2 papulopustular eruption compared to $55 \%$ in the placebo arm. Patients treated with tetracycline reported better quality of life scores (Skindex-16), including a decrease in skin burning and skin irritation.

\section{Novel Agents in the Management of EGFRI-Associated Skin Rash}

In addition to the preventive measures, topical and systemic treatments as described above, novel agents are being developed to reduce the severity and incidence of skin rash associated with EGFRI. One such agent is menadione, a synthetic prodrug of vitamin $\mathrm{K}$ [65]. When used topically/locally, menadione inhibits phosphatase and reduces the degree of EGFR inhibition induced by EGFRI on the skin, hence preventing the potential dermatological side effects from EGFRI's. It is currently undergoing phase I and II studies.

As a motivating factor, there is a clear positive correlation of efficacy and survival benefits with increasing severity of EGFRI-associated rash $[1,66]$. This strong association of rash with survival has led to studies investigating a dose escalation protocol titrated to rash such as the EVEREST study involving cetuximab dose escalation in metastatic colon cancer [67]. The rash and survival correlation also makes one think of the possible immune mechanism behind the rash and the tumor response, where a possible increase in 
systemic cytokines/chemokines results in immunomodulation at the tumor level resulting in a better response. Various biochemical compounds are under development to actually augment this immune response to be used in conjunction with the EGFRI therapy [68], most prominently Imprime PGG (Biothera) has shown to improve survival even in the KRAS mutant colorectal cancer patients when used in combination with cetuximab (Erbitux) $[69,70]$.

\section{Management of Radiation Dermatitis in Patients Receiving Anti-EGFR Monoclonal Antibodies}

Monoclonal antibodies against EGFR are increasingly used with external beam radiotherapy in the treatment of head and neck cancer patients. By itself, radiation dermatitis is an expected side effect of radiation therapy secondary to direct injury to the skin tissue in the treatment field. Epidermal basal cells and connective tissue damage can occur in the first couple of weeks of radiotherapy which can be compounded by the use of EGFR inhibitor therapy. It is important that multidisciplinary approach is taken in treating this rash planning and delivery of radiotherapy are important and if rashes are severe then consideration should be made to adjust the radiotherapy dose.

Cetuximab is the only targeted biological therapy approved in conjunction with radiotherapy for head and neck cancer. In the phase III trial that led to initial FDA approval for this indication, the frequency of observed radiation dermatitis (evaluated separately from acneiform dermatitis) was high (85-90\%) in both cetuximab-treated and untreated patients [71]. However, high-grade radiation dermatitis has been reported from the addition of cetuximab to radiotherapy [72], and higher severe (grade 3-4) in-field dermatitis has been observed when cetuximab has been combined with cisplatin-based chemoradiotherapy [73]. Radiation dermatitis ranges from erythema and dry or wet desquamation to skin necrosis or ulceration of full thickness dermis with spontaneous bleeding from the involved site. Current recommendations as established by the MASCC skin toxicity study group [62] state that the irradiated area should be kept clean and dry, even when ulcerated. Gentle washing and drying of the skin within the radiation field have been shown to reduce the acute radiation-induced skin reaction $[74,75]$. High-dose topical steroids have also been employed to treat radiation-induced dermatitis (mometasone, methylprednisolone, beclomethasone, and betamethasone topical preparations) $[76,77]$. It is imperative that the healthcare team take a proactive approach in preventing this complication and prescribe appropriate therapy to treat the local area either by topical or systemic therapy described above in a timely manner.

\section{Conclusion}

The EGFR inhibitors have become a valid antitumor therapy in many cancers and associated with their use is the commonly reported skin rash and other dermatologic toxicities.
The skin toxicity can impair quality of life and interfere with patient's compliance with therapy. With the advent of such agents and future approvals of newer EGFRIs in development, it is becoming exceedingly important for the healthcare team to not only recognize and treat these adverse effects to improve individual patient's quality of life and compliance but employ appropriate adjustment of dose of medication when needed. With the exciting new developments in the field, we do hope to uncover the best mode of management of these adverse effects and at the same time prevent attenuation of the immune response which could benefit the patient in terms of treatment efficacy and survival outcomes associated with EGFRI therapy.

\section{Acknowledgment}

This work is supported by NIH-K12 CA132783 Paul Calabresi Career Development Award for Clinical Oncology.

\section{References}

[1] R. Peréz-Soler and L. Saltz, "Cutaneous adverse effects with HER1/EGFR-targeted agents: is there a silver lining?" Journal of Clinical Oncology, vol. 23, no. 22, pp. 5235-5246, 2005.

[2] M. E. Lacouture, M. L. Maitland, S. Segaert et al., "A proposed EGFR inhibitor dermatologic adverse event-specific grading scale from the MASCC skin toxicity study group," Supportive Care in Cancer, vol. 18, no. 4, pp. 509-522, 2010.

[3] S. S. Joshi, S. Ortiz, J. N. Witherspoon et al., "Effects of epidermal growth factor receptor inhibitor-induced dermatologic toxicities on quality of life," Cancer, vol. 116, no. 16, pp. 39163923, 2010.

[4] R. S. Herbst, P. M. LoRusso, M. Purdom, and D. Ward, "Dermatologic side effects associated with gefitinib therapy: clinical experience and management," Clinical Lung Cancer, vol. 4, no. 6, pp. 366-369, 2003.

[5] M. J. Moore, D. Goldstein, J. Hamm et al., "Erlotinib plus gemcitabine compared with gemcitabine alone in patients with advanced pancreatic cancer: a phase III trial of the National Cancer Institute of Canada Clinical Trials Group," Journal of Clinical Oncology, vol. 25, no. 15, pp. 1960-1966, 2007.

[6] M. Fukuoka, S. Yano, G. Giaccone et al., "Multi-institutional randomized phase II trial of gefitinib for previously treated patients with advanced non-small-cell lung cancer," Journal of Clinical Oncology, vol. 22, no. 23, p. 4811, 2004.

[7] A. Scope, A. L. C. Agero, S. W. Dusza et al., "Randomized double-blind trial of prophylactic oral minocycline and topical tazarotene for cetuximab-associated acne-like eruption," Journal of Clinical Oncology, vol. 25, no. 34, pp. 5390-5396, 2007.

[8] M. E. Lacouture, E. P. Mitchell, B. Piperdi et al., "Skin Toxicity Evaluation Protocol With Panitumumab (STEPP), a phase II, open-label, randomized trial evaluating the impact of a preemptive skin treatment regimen on skin toxicities and quality of life in patients with metastatic colorectal cancer," Journal of Clinical Oncology, vol. 28, no. 8, pp. 1351-1357, 2010.

[9] K. Potthoff, R. Hofheinz, J. C. Hassel et al., "Interdisciplinary management of EGFR-inhibitor-induced skin reactions: a German expert opinion," Annals of Oncology, vol. 22, no. 3, pp. 524-535, 2011. 
[10] M. Duvic, "EGFR inhibitor-associated acneiform folliculitis: assessment and management," American Journal of Clinical Dermatology, vol. 9, no. 5, pp. 285-294, 2008.

[11] R. Pérez-Soler, A. Chachoua, L. A. Hammond et al., "Determinants of tumor response and survival with erlotinib in patients with non-small-cell lung cancer," Journal of Clinical Oncology, vol. 22, no. 16, pp. 3238-3247, 2004.

[12] R. S. Herbst, D. Prager, R. Hermann et al., "TRIBUTE: a phase III trial of erlotinib hydrochloride (OSI-774) combined with carboplatin and paclitaxel chemotherapy in advanced nonsmall-cell lung cancer," Journal of Clinical Oncology, vol. 23, no. 25, pp. 5892-5899, 2005.

[13] U. Gatzemeier, A. Pluzanska, A. Szczesna et al., "Phase III study of erlotinib in combination with cisplatin and gemcitabine in advanced non-small-cell lung cancer: the Tarceva lung cancer investigation trial," Journal of Clinical Oncology, vol. 25, no. 12, pp. 1545-1552, 2007.

[14] J. N. Rich, D. A. Reardon, T. Peery et al., "Phase II trial of gefitinib in recurrent glioblastoma," Journal of Clinical Oncology, vol. 22, pp. 133-142, 2004.

[15] B. Burtness, M. A. Goldwasser, W. Flood, B. Mattar, and A. A. Forastiere, "Phase III randomized trial of cisplatin plus placebo compared with cisplatin plus cetuximab in metastatic/recurrent head and neck cancer: an Eastern Cooperative Oncology Group Study," Journal of Clinical Oncology, vol. 23, no. 34, pp. 8646-8654, 2005.

[16] J. B. Vermorken, J. Trigo, R. Hitt et al., "Open-label, uncontrolled, multicenter phase II study to evaluate the efficacy and toxicity of cetuximab as a single agent in patients with recurrent and/or metastatic squamous cell carcinoma of the head and neck who failed to respond to platinum-based therapy," Journal of Clinical Oncology, vol. 25, no. 16, pp. 2171 2177, 2007.

[17] J. Marshall, "Clinical implications of the mechanism of epidermal growth factor receptors inhibitors," Cancer, vol. 107, no. 6, pp. 1207-1218, 2006.

[18] A. Okines, D. Cunningham, and I. Chau, "Targeting the human EGFR family in esophagogastric cancer," Nature Reviews Clinical Oncology, vol. 8, no. 8, pp. 492-503, 2011.

[19] R. S. Herbst and D. M. Shin, "Monoclonal antibodies to target epidermal growth factor receptor-positive tumors a new paradigm for cancer therapy," Cancer, vol. 94, no. 5, pp. 1593$1611,2002$.

[20] J. Mendelsohn, "The epidermal growth factor receptor as a target for cancer therapy," Endocrine-Related Cancer, vol. 8, no. 1, pp. 3-9, 2001.

[21] Y. Yarden and M. X. Sliwkowski, "Untangling the ErbB signalling network," Nature Reviews Molecular Cell Biology, vol. 2, no. 2, pp. 127-137, 2001.

[22] E. S. Kim, F. R. Khuri, and R. S. Herbst, "Epidermal growth factor receptor biology (IMC-C225)," Current Opinion in Oncology, vol. 13, no. 6, pp. 506-513, 2001.

[23] G. Carpenter and S. Cohen, "epidermal growth factor," Annual Review of Biochemistry, vol. 48, pp. 193-216, 1979.

[24] M. Yao, T. Shuin, H. Misaki, and Y. Kubota, "Enhanced expression of c-myc and epidermal growth factor receptor (C-erbB-1) genes in primary human renal cancer," Cancer Research, vol. 48, no. 23, pp. 6753-6757, 1988.

[25] B. W. Ennis, M. E. Lippman, and R. B. Dickson, "The EGF receptor system as a target for antitumor therapy," Cancer Investigation, vol. 9, no. 5, pp. 553-562, 1991.

[26] J. C. Hu, P. Sadeghi, L. C. Pinter-Brown, S. Yashar, and M. W. Chiu, "Cutaneous side effects of epidermal growth factor receptor inhibitors: clinical presentation, pathogenesis, and management," Journal of the American Academy of Dermatology, vol. 56, no. 2, pp. 317-326, 2007.

[27] L. E. King Jr., R. E. Gates, C. M. Stoscheck, and L. B. Nanney, "The EGF/TGF alpha receptor in skin," Journal of Investigative Dermatology, vol. 94, pp. 164S-170S, 1990.

[28] R. Sipples, "Common side effects of anti-EGFR therapy: acneform rash," Seminars in Oncology Nursing, vol. 22, no. 1, pp. 28-34, 2006.

[29] E. Guttman-Yassky, A. Mita, M. De Jonge et al., "Characterisation of the cutaneous pathology in non-small cell lung cancer (NSCLC) patients treated with the EGFR tyrosine kinase inhibitor erlotinib," European Journal of Cancer, vol. 46, no. 11, pp. 2010-2019, 2010.

[30] S. Kobayashi, T. J. Boggon, T. Dayaram et al., "EGFR mutation and resistance of non-small-cell lung cancer to gefitinib," The New England Journal of Medicine, vol. 352, no. 8, pp. 786-792, 2005.

[31] S. Parmar, C. Schumann, R. üdiger S et al., "Pharmacogenetic predictors for EGFR-inhibitor-associated skin toxicity," The Pharmacogenomics Journal. In press.

[32] K. Klinghammer, M. Knödler, A. Schmittel, V. Budach, U. Keilholz, and I. Tinhofer, "Association of epidermal growth factor receptor polymorphism, skin toxicity, and outcome in patients with squamous cell carcinoma of the head and neck receiving cetuximab-docetaxel treatment," Clinical Cancer Research, vol. 16, no. 1, pp. 304-310, 2010.

[33] C. L. Huang, C. H. Yang, K. H. Yeh et al., "EGFR intron 1 dinucleotide repeat polymorphism is associated with the occurrence of skin rash with gefitinib treatment," Lung Cancer, vol. 64, no. 3, pp. 346-351, 2009.

[34] C. M. Rudin, W. Liu, A. Desai et al., "Pharmacogenomic and pharmacokinetic determinants of erlotinib toxicity," Journal of Clinical Oncology, vol. 26, no. 7, pp. 1119-1127, 2008.

[35] L. I. Wagner and M. E. Lacouture, "Dermatologic toxicities associated with EGFR inhibitors: the clinical psychologist's perspective. Impact on health-related quality of life and implications for clinical management of psychological sequelae," Oncology, vol. 21, no. 11, pp. 34-36, 2007.

[36] B. You, A. Brade, J. M. Magalhaes et al., "A dose-escalation phase I trial of nimotuzumab, an antibody against the epidermal growth factor receptor, in patients with advanced solid malignancies," Investigational New Drugs, vol. 29, pp. 996-1003, 2010.

[37] F. Rojo, E. Gracias, N. Villena et al., "Pharmacodynamic trial of nimotuzumab in unresectable squamous cell carcinoma of the head and neck: a SENDO Foundation study," Clinical Cancer Research, vol. 16, no. 8, pp. 2474-2482, 2010.

[38] M. O. Rodríguez, T. C. Rivero, R. D. C. Bahi et al., "Nimotuzumab plus radiotherapy for unresectable squamous-cell carcinoma of the head and neck," Cancer Biology and Therapy, vol. 9, no. 5, pp. 343-349, 2010.

[39] A. Wollenberg, N. Moosmann, E. Klein, and K. Katzer, "A tool for scoring of acneiform skin eruptions induced by EGF receptor inhibition," Experimental Dermatology, vol. 17, no. 9, pp. 790-792, 2008.

[40] R. Pérez-Soler, J. P. Delord, A. Halpern et al., "HER1/EGFR inhibitor-associated rash: future directions for management and investigation outcomes from the HER1/EGFR inhibitor rash management forum," Oncologist, vol. 10, no. 5, pp. 345356, 2005 .

[41] M. E. Lacouture, M. L. Maitland, S. Segaert et al., "A proposed EGFR inhibitor dermatologic adverse event-specific grading 
scale from the MASCC skin toxicity study group," Supportive Care in Cancer, vol. 18, no. 4, pp. 509-522, 2010.

[42] A. F. S. Galimont-Collen, L. E. Vos, A. P. M. Lavrijsen, J. Ouwerkerk, and H. Gelderblom, "Classification and management of skin, hair, nail and mucosal side-effects of epidermal growth factor receptor (EGFR) inhibitors," European Journal of Cancer, vol. 43, no. 5, pp. 845-851, 2007.

[43] E. P. Mitchell, R. Perez-Soler, E. Van Cutsem, and M. E. Lacouture, "Clinical presentation and pathophysiology of EGFRI dermatologic toxicities,” Oncology, vol. 21, no. 11, pp. 4-9, 2007.

[44] J. Boucher, L. Olson, and B. Piperdi, "Preemptive management of dermatologic toxicities associated with epidermal growth factor receptor inhibitors," Clinical Journal of Oncology Nursing, vol. 15, pp. 501-508, 2011.

[45] T. J. Lynch Jr., E. S. Kim, B. Eaby, J. Garey, D. P. West, and M. E. Lacouture, "Epidermal growth factor receptor inhibitorassociated cutaneous toxicities: an evolving paradigm in clinical management," Oncologist, vol. 12, no. 5, pp. 610-621, 2007.

[46] M. E. Lacouture and S. E. Lai, “The PRIDE (Papulopustules and/or paronychia, Regulatory abnormalities of hair growth, Itching, and Dryness due to Epidermal growth factor receptor inhibitors) syndrome," British Journal of Dermatology, vol. 155, no. 4, pp. 852-854, 2006.

[47] S. Basti, "Ocular toxicities of epidermal growth factor receptor inhibitors and their management," Cancer Nursing, vol. 30, no. 4, pp. S10-S16, 2007.

[48] R. Gutzmer, J. C. Becker, A. Enk et al., "Management of cutaneous side effects of EGFR inhibitors: recommendations from a German expert panel for the primary treating physician," Journal of the German Society of Dermatology, vol. 9, no. 3, pp. 195-203, 2011.

[49] C. Gridelli, P. Maione, D. Amoroso et al., "Clinical significance and treatment of skin rash from erlotinib in non-small cell lung cancer patients: results of an Experts Panel Meeting," Critical Reviews in Oncology/Hematology, vol. 66, no. 2, pp. 155-162, 2008.

[50] J. Bernier, J. Bonner, J. B. Vermorken et al., "Consensus guidelines for the management of radiation dermatitis and coexisting acne-like rash in patients receiving radiotherapy plus EGFR inhibitors for the treatment of squamous cell carcinoma of the head and neck," Annals of Oncology, vol. 19, no. 1, pp. 142-149, 2008.

[51] E. H. Tan and A. Chan, "Evidence-based treatment options for the management of skin toxicities associated with epidermal growth factor receptor inhibitors," Annals of Pharmacotherapy, vol. 43, no. 10, pp. 1658-1666, 2009.

[52] A. Jatoi, "Sunshine and rash: testing the role of sunscreen to prevent epidermal growth factor receptor inhibitor-induced rash," Supportive Cancer Therapy, vol. 4, no. 4, pp. 198-202, 2007.

[53] V. Hirsh, "Managing treatment-related adverse events associated with EGFR tyrosine kinase inhibitors in advanced nonsmall-cell lung cancer," Current Oncology, vol. 18, no. 3, pp. 126-138, 2011.

[54] L. B. Saltz, N. J. Meropol, P. J. Loehrer, M. N. Needle, J. Kopit, and R. J. Mayer, "Phase II trial of cetuximab in patients with refractory colorectal cancer that expresses the epidermal growth factor receptor," Journal of Clinical Oncology, vol. 22, no. 7, pp. 1201-1208, 2004.

[55] D. Cunningham, Y. Humblet, S. Siena et al., "Cetuximab monotherapy and cetuximab plus irinotecan in irinotecan- refractory metastatic colorectal cancer," The New England Journal of Medicine, vol. 351, no. 4, pp. 337-345, 2004.

[56] E. Van Cutsem, M. Peeters, S. Siena et al., "Open-label phase III trial of panitumumab plus best supportive care compared with best supportive care alone in patients with chemotherapy- refractory metastatic colorectal cancer," Journal of Clinical Oncology, vol. 25, no. 13, pp. 1658-1664, 2007.

[57] D. Soulieres, N. N. Senzer, E. E. Vokes, M. Hidalgo, S. S. Agarvala, and L. L. Siu, "Multicenter phase II study of erlotinib, an oral epidermal growth factor receptor tyrosine kinase inhibitor, in patients with recurrent or metastatic squamous cell cancer of the head and neck," Journal of Clinical Oncology, vol. 22, no. 1, pp. 77-85, 2004.

[58] M. G. Kris, R. B. Natale, R. S. Herbst et al., "Efficacy of gefitinib, an inhibitor of the epidermal growth factor receptor tyrosine kinase, in symptomatic patients with non-small cell lung cancer: a randomized trial," Journal of the American Medical Association, vol. 290, no. 16, pp. 2149-2158, 2003.

[59] C. E. Geyer, J. Forster, D. Lindquist et al., "Lapatinib plus capecitabine for HER2-positive advanced breast cancer," The New England Journal of Medicine, vol. 355, no. 26, pp. 27332743, 2006.

[60] S. Segaert and E. Van Cutsem, "Clinical signs, pathophysiology and management of skin toxicity during therapy with epidermal growth factor receptor inhibitors," Annals of Oncology, vol. 16, no. 9, pp. 1425-1433, 2005.

[61] S. Segaert, J. Tabernero, O. Chosidow et al., "The management of skin reactions in cancer patients receiving epidermal growth factor receptor targeted therapies," Journal of the German Society of Dermatology, vol. 3, no. 8, pp. 599-606, 2005.

[62] M. E. Lacouture, M. J. Anadkat, R. J. Bensadoun et al., "Clinical practice guidelines for the prevention and treatment of EGFR inhibitor-associated dermatologic toxicities," Supportive Care in Cancer, vol. 19, pp. 1079-1095, 2011.

[63] A. Jatoi, K. Rowland, J. A. Sloan et al., "Tetracycline to prevent epidermal growth factor receptor inhibitor-induced skin rashes: results of a placebo-controlled trial from the North Central Cancer Treatment Group (N03CB)," Cancer, vol. 113, no. 4, pp. 847-853, 2008.

[64] A. Jatoi, S. R. Dakhil, J. A. Sloan et al., "Prophylactic tetracycline does not diminish the severity of epidermal growth factor receptor (EGFR) inhibitor-induced rash: results from the North Central Cancer Treatment Group (Supplementary N03CB)," Supportive Care in Cancer, vol. 25, article 494s, 2010.

[65] R. Perez-Soler, Y. Zou, T. Li, and Y. H. Ling, "The phosphatase inhibitor menadione (vitamin K3) protects cells from EGFR inhibition by erlotinib and cetuximab," Clinical Cancer Research, vol. 17, pp. 6766-6777, 2011.

[66] B. Wacker, T. Nagrani, J. Weinberg, K. Witt, G. Clark, and P. J. Cagnoni, "Correlation between development of rash and efficacy in patients treated with the epidermal growth factor receptor tyrosine kinase inhibitor erlotinib in two large phase III studies," Clinical Cancer Research, vol. 13, no. 13, pp. 39133921, 2007.

[67] B. Klein and M. Gottfried, "Targeted agents to improve treatment results in colon cancer: bevacizumab and cetuximab," Journal of B.U.ON., vol. 12, supplement 1, pp. S127-S136, 2007.

[68] F. Hong, R. D. Hansen, J. Yan et al., " $\beta$-glucan functions as an adjuvant for monoclonal antibody immunotherapy by recruiting tumoricidal granulocytes as killer cells," Cancer Research, vol. 63, no. 24, pp. 9023-9031, 2003.

[69] M. E. Tamayo GHC, J. B. Bautista, M. L. Flores et al., "A phase $\mathrm{Ib} / 2$, dose-escalating, safety, and efficacy study of imprime 
PGG, cetuximab and irinotecan in patients with advanced colorectal cancer (CRC)," Journal of Clinical Oncology, vol. 28, abstract e14103, 2010, ASCO Annual Meeting Proceedings.

[70] N. H. Segal NS, P. Gada, L. M. Marsh, M. A. Gargano, and M. L. Patchen LS, "Imprime PGG improves survival in KRAS mutant colorectal cancer patients," in European Society for Medical Oncology (ESMO) 13th World Congress on Gastrointestinal Cancer, 2011.

[71] J. A. Bonner, P. M. Harari, J. Giralt et al., "Radiotherapy plus cetuximab for locoregionally advanced head and neck cancer: 5 -year survival data from a phase 3 randomised trial, and relation between cetuximab-induced rash and survival," The Lancet Oncology, vol. 11, no. 1, pp. 21-28, 2010.

[72] W. Budach, E. Bölke, and B. Homey, "Severe cutaneous reaction during radiation therapy with concurrent cetuximab," The New England Journal of Medicine, vol. 357, no. 5, pp. 514515, 2007.

[73] K. Ang, Q. Zhang, D. Rosenthal et al., "A randomized phase III trial (RTOG 0522) of concurrent accelerated radiation plus cisplatin with or without cetuximab for stage III-IV head and neck squamous cell carcinomas (HNC)," Journal of Clinical Oncology, vol. 29, p. 5500, 2011.

[74] I. R. Campbell and M. H. Illingworth, "Can patients wash during radiotherapy to the breast or chest wall? A randomized controlled trial," Clinical Oncology, vol. 4, no. 2, pp. 78-82, 1992.

[75] I. Roy, A. Fortin, and M. Larochelle, "The impact of skin washing with water and soap during breast irradiation: a randomized study," Radiotherapy and Oncology, vol. 58, no. 3, pp. 333-339, 2001.

[76] N. Salvo, E. Barnes, J. van Draanen et al., "Prophylaxis and management of acute radiation-induced skin reactions: a systematic review of the literature," Current Oncology, vol. 17, no. 4, pp. 94-112, 2010.

[77] R. C. Miller, D. J. Schwartz, J. A. Sloan et al., "Mometasone furoate effect on acute skin toxicity in breast cancer patients receiving radiotherapy: a phase III double-blind, randomized trial from the North Central Cancer Treatment Group N06C4," International Journal of Radiation Oncology Biology Physics, vol. 79, no. 5, pp. 1460-1466, 2011. 


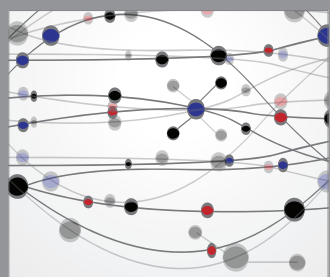

The Scientific World Journal
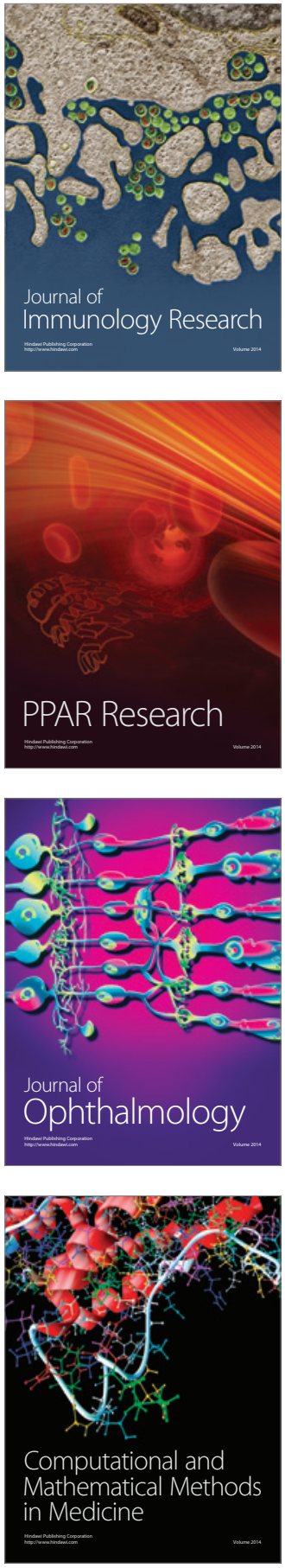

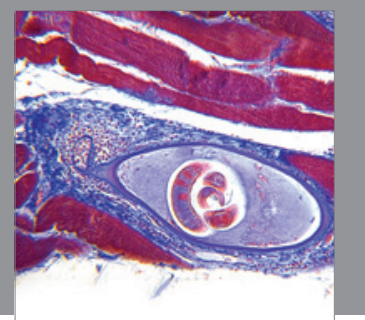

Gastroenterology

Research and Practice
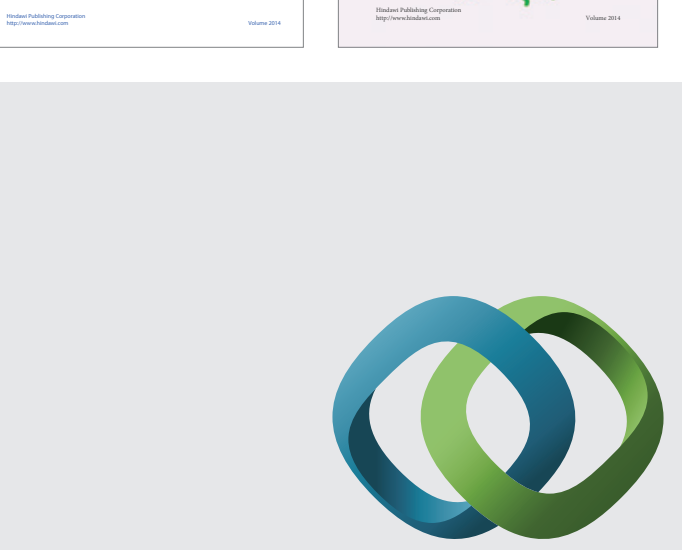

\section{Hindawi}

Submit your manuscripts at

http://www.hindawi.com
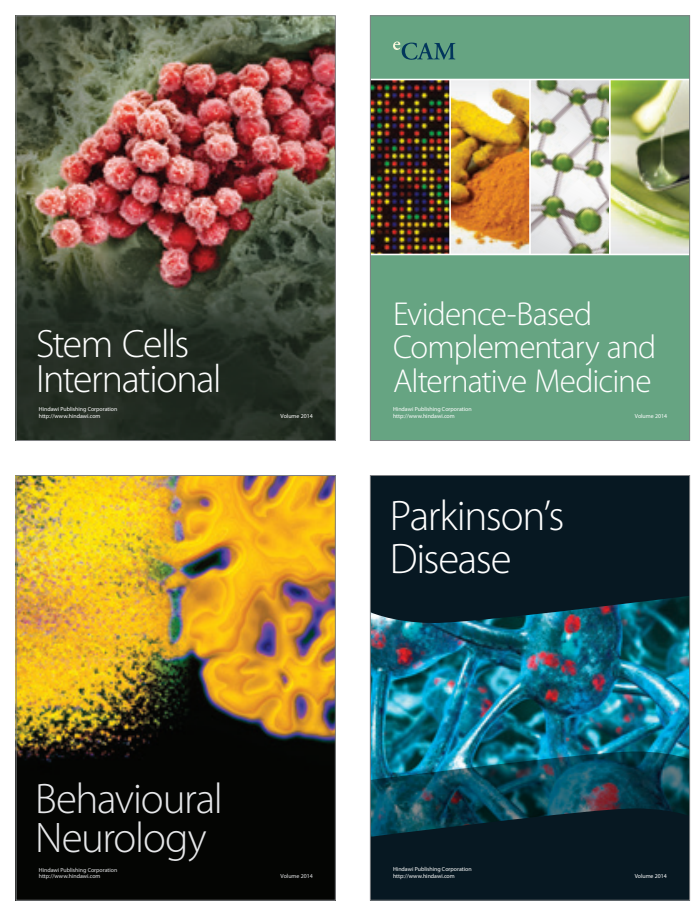

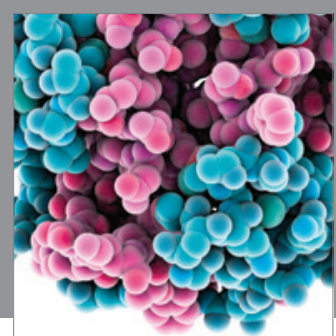

Journal of
Diabetes Research

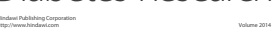

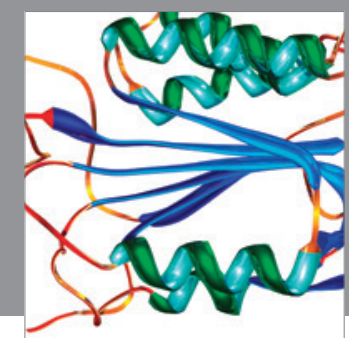

Disease Markers
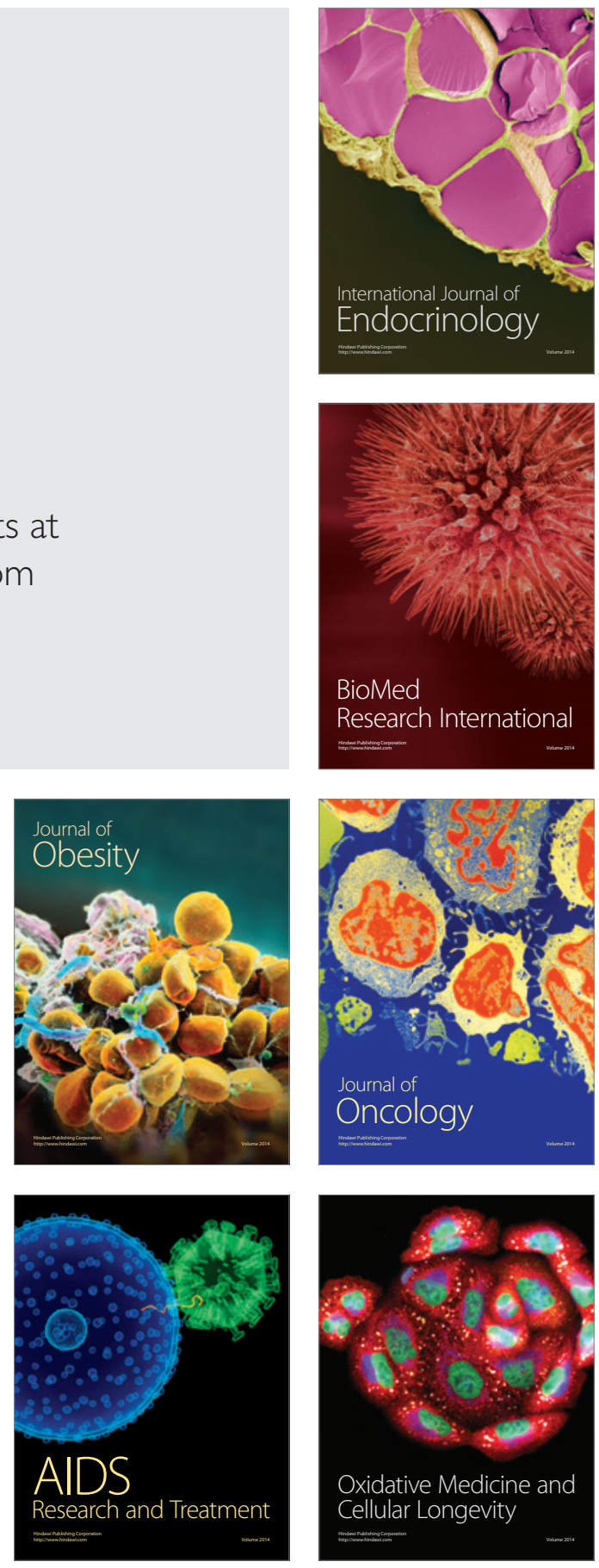